\title{
Using Psychophysiological Measurements in Physically Demanding Virtual Environments
}

\author{
Domen Novak, Matjaž Mihelj, and Marko Munih \\ University of Ljubljana, Faculty of Electrical Engineering, \\ Tržaška c. 25, 1000 Ljubljana, Slovenia \\ domen.novak@robo.fe.uni-lj.si
}

\begin{abstract}
Psychophysiological evaluation of mental workload in humancomputer interaction has generally been limited to situations with little physical load. This paper examines the viability of using heart rate, skin conductance, respiration and peripheral skin temperature as psychophysiological indicators in a physically demanding task performed in a simple virtual environment. Respiratory rate was found to be a good indicator of arousal while respiratory rate variability and skin temperature indicated changes in valence.
\end{abstract}

Keywords: Affective HCI, Human Factors, Multi-Modal Interfaces.

\section{Introduction}

Human-computer interaction makes use of findings from many scientific fields to study the response of humans to technology. One such field is psychophysiology, which describes how a person's physiological responses reflect his or her psychological state. Physiological responses are a valuable tool for human-computer research since they provide an objective estimate of the user's psychological state. The most commonly used responses are those of the autonomic nervous system: heart rate, skin conductance, respiration and skin temperature. Unfortunately, they are not only influenced by a person's psychological state, but also by any physical activity. Thus, many studies of psychophysiological responses try to limit physical activity to a minimum. However, to some degree, responses of the autonomic nervous system may provide meaningful results even in physically demanding environments. Our goal was to determine which autonomic nervous system responses could be used to determine the level of mental activity, stress and frustration in tasks that require physical load.

\section{Materials and Methods}

This paper presents a study of a physically demanding hand-eye coordination task performed with different difficulty levels. Thirty healthy subjects (age 19-46 years, mean 26.2, 23 male, 7 female) participated in the study. They were presented with the classic inverted pendulum problem. An unstable pole standing atop a cart was shown on the screen, and participants had to balance the pole (pendulum) by moving the cart using a HapticMaster haptic interface (manufactured by Moog FCS) that also measured the 
force exerted. The HapticMaster actively resisted movement, requiring a significant force to move. If the pole fell to a horizontal position, it was reset to a nearly vertical position. Figure 1 shows a participant balancing the pendulum.

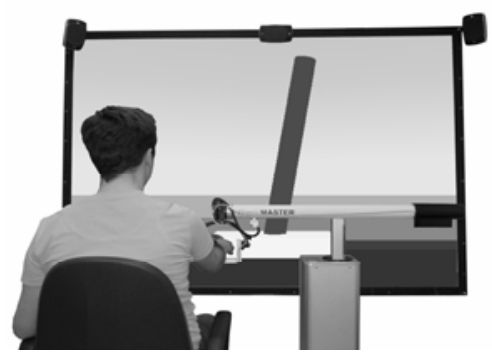

Fig. 1. Participant performing the pendulum task

Three difficulty levels of the pendulum task (PT) were implemented. While the medium difficulty level (PT.M) was moderately challenging and the easy level (PT.E) was only slightly easier than the medium level, the hard level (PT.H) was intended to be difficult to the point of frustration. This was done by subtly manipulating the mathematical model describing the cart and pole in order to make the pole much less responsive to user input. In addition to the PT, a control task (CT) was introduced to evaluate the effect of physical load in the absence of mental load. During the CT, participants had to move the HapticMaster left and right at an even, moderate speed while nothing was shown on the screen. Participants were informed that they would need to perform the PT three times, but that there would be no change in difficulty.

The participants rested for five minutes in order to obtain baseline values. Afterwards, CT, PT.E, PT.M and PT.H were performed in random order for five minutes each. After each period, participants were presented with nine-point arousal and valence self-report scales from the self-assessment manikin [1].

The electrocardiogram was recorded using surface electrodes affixed to the chest and abdomen. Skin conductance was measured from the second and third fingers of the non-dominant hand using a g.GSR sensor (g.tec Medical Engineering GmbH). Respiratory rate was obtained using a thermistor-based SleepSense Flow sensor. Skin temperature was measured using a g.TEMP sensor (g.tec) attached to the distal phalanx of the fifth finger. All signals were sampled at $2.4 \mathrm{kHz}$. After the experiment, the following physiological parameters were calculated for each time period: mean heart rate (HR), two standardized measures of heart rate variability (SDNN and RMSSD [2]), mean skin conductance level (SCL), nonspecific skin conductance response frequency $(\mathrm{SCRF})$, mean respiratory rate $(\mathrm{RR})$, respiratory rate variance (RRV) and final skin temperature (ST). In addition to physiological measurements, the mean absolute force exerted by participants was calculated for each time period.

The purpose of the study was to determine how physiological responses are affected by psychological arousal and valence in the presence of physical load. Arousal is defined as general mental activity while valence indicates whether the person's emotions are positive or negative [3]. Comparing PT.M to CT was expected to show the effects of arousal. Since PT.H was designed to be frustratingly difficult, comparing it to PT.M was 
expected to primarily show the effects of negative valence. Significance was evaluated using a one-way repeated-measures ANOVA followed by the Tukey test in post-hoc analysis. Differences were significant for $\mathrm{p}<0.05$.

\section{Results and Discussion}

Results of the self-assessment manikin showed that self-reported arousal was higher during CT than during the baseline period $(\mathrm{p}=0.02)$. Thus, we cannot rule out some influence of arousal during CT. Arousal during all three difficulty levels of PT was higher than during CT $(\mathrm{p}<0.001)$. There were no significant differences in arousal between difficulty levels. Self-reported valence decreased from baseline during CT (p $=0.02)$ and PT.H $(\mathrm{p}=0.007)$. Valence was lower during PT.H than during the other two difficulty levels ( $\mathrm{p}<0.01$ for both comparisons). It was also lower during CT than during PT.E and PT.M ( $\mathrm{p}<0.05$ for both comparisons).

Mean absolute force during $\mathrm{CT}$ was more than twice as high as during all three difficulty levels of PT $(\mathrm{p}<0.001)$. It was also higher during PT.H than PT.E and PT.M ( $p<0.05$ for both comparisons). Thus, we must be cautious when comparing physiological responses. The change in a physiological response may not have been caused by changes in psychological state, but by the differences in physical load.

The following physiological parameters changed significantly from baseline to CT: HR (increased by $10.2 \%, \mathrm{p}<0.001$ ), SDNN (incr. by $17.1 \%, \mathrm{p}<0.01$ ), RMSSD (incr. by $23.9 \%, \mathrm{p}<0.01$ ), SCL (incr. by $1 \mu \mathrm{S}, \mathrm{p}<0.001$ ), SCRF (incr. by $236.2 \%$, p $<0.001$ ), RR (incr. by 17.4\%, p < 0.001) and RRV (decreased by 6\%, p = 0.04). Thus, all physiological parameters other than ST were affected by physical load.

The following physiological parameters were significantly different between CT and PT.M: HR (lower in PT.M, p < 0.001), RMSSD (lower in PT.M, p < 0.001), RR (higher in PT.M, $\mathrm{p}=0.006$ ) and RRV (lower in PT.M, $\mathrm{p}=0.02$ ). The difference in SCRF approached significance (higher in PT.M, $\mathrm{p}=0.07$ ).

Since there was no significant difference in SCL between CT and PT.M, it appeared to be primarily affected by physical load and thus not useful in physically demanding tasks. Previous studies have noted a connection between SCRF and arousal [4]. However, since the difference in SCRF between CT and PT.M was not quite significant, SCRF is apparently only a reliable indicator of arousal if little physical load is involved. On the other hand, since SCRF increases with physical load, we can assume that it would have been much lower in CT if the mean absolute force exerted had been the same as in PT.M. In that case, the difference between CT and PT.M probably would have been significant. RR during CT was significantly higher than the baseline value, but RR during PT.M was higher still. Since mean absolute force was higher during $\mathrm{CT}$, the changes in RR cannot be attributed solely to changes in physical load, but must be caused by arousal. Previous studies have indeed shown respiratory rate to be connected to arousal [5]. Similarly, RRV during CT was significantly lower than the baseline value, but was even lower during PT.M. Mental arousal thus apparently also decreases RRV. By far the highest increase in HR was during CT, where the exerted force was also the highest. Since there was no significant difference between difficulty levels, we can conclude that HR was mostly influenced by physical load. Similarly, though significant differences in SDNN and 
RMSSD were observed between CT and PT.M, none can be reliably attributed to changes in psychological state - they may have been caused by physical load.

No physiological parameters showed significant differences between PT.E and PT.M. There were two significant differences between PT.M and PT.H: RRV was lower during PT.M ( $<<0.001)$ while ST was higher during PT.M ( $\mathrm{p}=0.01)$.

Differences between CT and PT.M indicated that RRV decreases as arousal increases. The difference in RRV between PT.M and PT.H suggests that RRV also increases as valence decreases (i.e. as frustration increases). Since ST significantly decreased from baseline only during PT.H and was lower during PT.H than during PT.M, it apparently decreases as valence decreases. Other studies have linked decreases in fingertip temperature to anxiety and stress [6], supporting our findings. Again, it is worth noting that ST was the only psychophysiological response not influenced by physical load.

\section{Conclusions}

We demonstrated a significant influence of both mental arousal and emotional valence on skin conductance, respiration and skin temperature even in the presence of moderate physical load. However, our study only confirms the usefulness of psychophysiological responses up to a certain level of physical load. Strenuous physical activity would probably cause physiological responses that would completely overshadow the physiological responses caused by changes in psychological state.

Acknowledgments. The work was funded by the EU Information and Communication Technologies Collaborative Project MIMICS grant 215756.

\section{References}

1. Lang, P.J.: Behavioral treatment and bio-behavioral assessment: Computer applications. In: Sidowski, J.B., Johnson, J.H., Williams, T.A. (eds.) Technology in Mental Health Care Delivery Systems, pp. 119-137. Abbex, Norwood (1980)

2. Task Force of the European Society of Cardiology and the North American Society of Pacing and Electrophysiology: Heart rate variability: Standards of measurement, physiological interpretation, and clinical use. European Heart Journal 17, 354-381 (1996)

3. Russell, J.A.: A circumplex model of affect. Journal of Personality and Social Psychology 39, 1161-1178 (1980)

4. Nikula, R.: Psychological correlates of nonspecific skin conductance responses. Psychophysiology 28, 86-90 (1991)

5. Brookings, J.B., Wilson, G.F., Swain, C.R.: Psychophysiological responses to changes in workload during simulated air traffic control. Biological Psychology 42, 361-377 (1996)

6. Min, B.C., et al.: Autonomic responses of young passengers contingent to the speed and driving mode of a vehicle. International Journal of Industrial Ergonomics 29, 187-198 (2002) 